\title{
UNIQUE FACTORIZATION IN RANDOM VARIABLES
}

\author{
BY F. ALBERTO GRÜNBAUM1 \\ Communicated June 30, 1975
}

The problem of determining the potential $q(x)$ from "spectral data" for the equation

$$
-y^{\prime \prime}(x)+q(x) y(x)=\lambda y(x), \quad-\infty<x<\infty,
$$

has been studied extensively. For a review, see [1] and [5].

A typical kind of result, associated with the names of Gelfand and Levitan tells you that if the discrete spectrum, the normalizing constants, and the reflection coefficient $R(k)$ are known, then $q(x)$ can, in principle, be determined uniquely.

Here we consider a random version of this problem. We envisage a situation where one keeps records of "spectral data" for noisy versions of the potential $q(x)$ and attempts to determine the "mean potential" from the distribution of the data.

It turns out that in this random case a smaller set of quantitites-measured over and over again-give a lot of information about $q(x)$. A similar situation develops in a variety of different setups (see, for instance, [3] and [4]).

Let $-\nabla^{2}$ stand for the $n \times n$ matrix

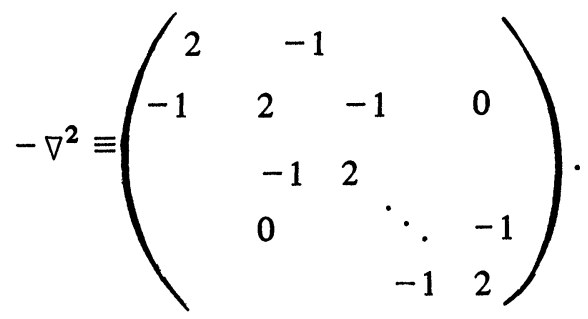

THEOREM I. Let $q_{1}, \ldots, q_{n}$ be independent Gaussian random variables with unknown means $\bar{q}_{1}, \ldots, \bar{q}_{n}$ and variances all equal to one. Then the joint distribution function of $\operatorname{tr}\left(-\nabla^{2}+q I\right)^{k}, k=1, \ldots, n$, determines the vector $\bar{q}_{1}, \ldots, \bar{q}_{n}$ up to a global reflection $\bar{q}_{i}^{\prime} \equiv \bar{q}_{n-i+1}$.

The theorem above says that the spectrum determines the potential up to a trivial reflection. This is to be compared with the nonrandom case where, in $60 \mathrm{G} 15$.

AMS (MOS) subject classifications (1970). Primary 62H05, 62H10, 10A25; Secondary

Key words and phrases. Inverse spectral problem, Gaussian variables.

1 Partially supported by NSF Grant MPS75-05576.

Copyright @ 1976, American Mathematical Society 
general, $n$ ! choices of potential are compatible with a given spectrum. For results of this kind, see [2].

The last remark indicates that one can guess how does the typical situation look by "ignoring" the off diagonal elements in $-\nabla^{2}+q I$. This gives a link between the two theorems below.

MAIN THEOREM. Let $X_{1}, X_{2}, \ldots, X_{n}$ be independent Gaussian random variables with variance one and unknown means $a_{1}, \ldots, a_{n}$. Then the distribution function of the product $Z=X_{1} \cdots X_{n}$ suffices to determine the product $a_{1} a_{2} \cdots a_{n}$ and the quantities $a_{1}^{2}, a_{2}^{2}, \cdots, a_{n}^{2}$ up to order.

THEOREM II. Under the conditions of Theorem I, the distribution function of $\operatorname{det}\left(-\nabla^{2}+q I\right)$ determines $\bar{q}$ up to an even number of changes of sign and a global reflection.

We give below a proof of the main theorem, which gives its title to this note, in the first nontrivial case, i.e., $n=4$. First, some remarks.

I. The Gaussian character of the $X_{i}$ 's is only a convenient device to get an easier proof. Actually if the $\left(X_{i}-a_{i}\right)$ 's have a symmetric distribution with second moment $\mu_{2}$ and fourth moment $\mu_{4}$, a condition like $\mu_{2} / \sqrt{ } 2>3 \mu_{2}^{2}-\mu_{4}$ suffices to make the proof below work. In the Gaussian case the right-hand side vanishes and our conditions say that we are in the truly random case $\mu_{2}>0$.

II. There is no need to assume that the distribution functions of the $\left(X_{i}-a_{i}\right)$ 's are the same. It is also unnecessary to assume these distributions are known in advance. In this way one gets

COROLlARY. From measurements of the volume of (slightly defective) replicas of a parallelogram, one can infer its length, width and height up to order.

Proof of THE MAIN THEOREM $(n=4)$. For simplicity, take all the means $a_{1}, \ldots, a_{4}$ to be nonzero. Introduce the elementary symmetric functions in the unknowns $a_{1}^{2}, a_{2}^{2}, a_{3}^{2}, a_{4}^{2}$, i.e.,

$$
\begin{aligned}
& \sigma_{1}=a_{1}^{2}+a_{2}^{2}+a_{3}^{2}+a_{4}^{2}, \quad \sigma_{2}=\sum_{i<j} a_{i}^{2} a_{j}^{2}, \\
& \sigma_{3}=\sum_{i<j<k} a_{i}^{2} a_{j}^{2} a_{k}^{2}, \quad \sigma_{4}=a_{1}^{2} a_{2}^{2} a_{3}^{2} a_{4}^{2} .
\end{aligned}
$$

The first three moments of the random variable $Z$ give

$$
\begin{aligned}
& Z_{1}=a_{1} a_{2} a_{3} a_{4}=\sigma_{4}^{1 / 2}, \quad Z_{2}=\sigma_{4}+\sigma_{3}+\sigma_{2}+\sigma_{1}+1, \\
& Z_{3}=\left(\sigma_{4}+3 \sigma_{3}+3^{2} \sigma_{2}+3^{3} \sigma_{1}+3^{4}\right) Z_{1} .
\end{aligned}
$$

The fourth moment $Z_{4}$ is a quadratic function of the $\sigma_{i}$ 's which is best written in terms of the new variables 


$$
\begin{gathered}
x=\frac{1}{\sqrt{38}}\left(3 \sigma_{1}-18 \sigma_{2}+\sigma_{3}\right), \quad y=\frac{1}{\sqrt{19}}\left(9 \sigma_{1}+3 \sigma_{2}+3 \sigma_{3}\right), \\
z=\frac{1}{\sqrt{2}}\left(3 \sigma_{1}-\sigma_{3}\right) .
\end{gathered}
$$

We have

$$
Z_{4}=19 y^{2}-12 z^{2}+y\left(C_{1} \sigma_{4}+C_{2}\right)+z\left(C_{3} \sigma_{4}+C_{4}\right)+\sigma_{4}^{2}+C_{5} \sigma_{4}
$$

with $C_{1}, C_{2}>0$.

Now from $Z_{1}, Z_{2}$ and $Z_{3}$, one can read off both $\sigma_{4}$ and $z$. Then $Z_{4}$ leads to two possible choices of $y$, only one of which can be positive since $C_{1}$ and $C_{2}$ are positive. But $y$ is positive by definition and thus can be determined from $Z_{4}$ and then used along with $z$ to get $x$ from $Z_{2}$. We have thus obtained $\sigma_{1}, \sigma_{2}, \sigma_{3}$, $\sigma_{4}$ from $Z_{1}, Z_{2}, Z_{3}, Z_{4}$ and the proof is finished.

\section{REFERENCES}

1. L. D. Faddeev, The inverse problem in the quantum theory of scattering, Uspehi Mat. Nauk 14 (1959), no. 4 (88), 57-119; English transl., J. Mathematical Phys. 4 (1963), 72-104. MR 22 \#1344; 26 \#7328.

2. S. Friedland, Matrices with prescribed offdiagonal elements, Israel J. Math. 11 (1972), 184-189.

3. F. A. Grünbaum, Inverse problems for nonlinear random systems, Partial Differential Equations and Related Topics, Lecture Notes in Math., vol. 446, Springer-Verlag, Berlin and New York, 1975, pp. 247-263.

4. - A remark on the phase problem in crystallography, Proc. Nat. Acad. Sci. U.S.A. 72 (1975), 1699-1701.

5. O. H. Hald, On discrete and numerical inverse Sturm-Liouville problems, Ph. D. Thesis, N. Y. U., 1972, Available from Dept. of Computer Sciences, Uppsala University, Sweden, Report 42, 1972.

\section{DEPARTMENT OF MATHEMATICS, UNIVERSITY OF CALIFORNIA, BERKELEY, CALIFORNIA 94720}

